\title{
Chemical characterization of atmospheric transported polycyclic aromatic hydrocarbons in Peninsular Malaysia: a quarter century view
}

\begin{abstract}
Atmosphere has long been known as free way for the transport of particle reactive chemicals far distances. Polycyclic aromatic hydrocarbons (PAHs) are priority pollutants in the environment where anthropogenic sources such as petroleum are studied. Chemical characterization of deposited PAH in Peninsular Malaysia showed that compounds resulting from combustion contribute more to atmospherically transported compounds than do uncombusted materials, which usually follow the lateral transport, local oil discharge and in-situ natural production. The composition and characterization of PAH demonstrated a higher abundance of parent compounds than of alkyl substitutes. These include pyrene, benzo(e)pyrene, benzo(a)pyrene and benzo(k)fluorenthene. Parent compounds represent a higher proportion of combusted organic materials as well as of petroleum that transfers via atmospheric movement rather than laterally via, for example, rivers and run-off discharges. Diagnostic chemical ratios of chemical compounds such as phenantherene to antheracene and fluorenthene to pyrene showed that combusted materials are dominant that are transferred by atmosphere movement, either over short or long distances, in the studied area.
\end{abstract}

Keyword: Atmosphere; PAHs; Petroleum; Combustion; Peninsular Malaysia 